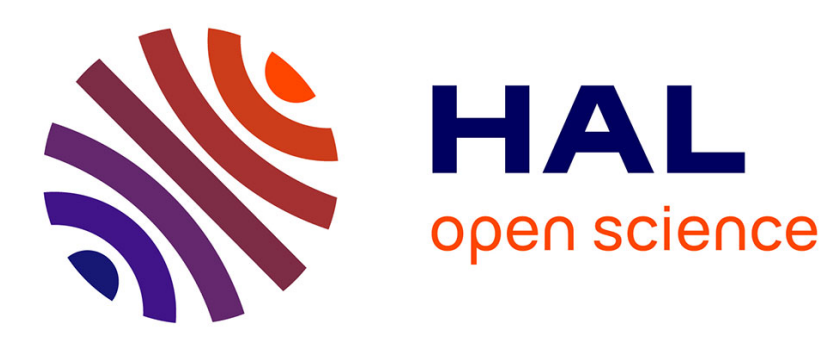

\title{
Minimization problems for eigenvalues of the Laplacian
}

Antoine Henrot

\section{To cite this version:}

Antoine Henrot. Minimization problems for eigenvalues of the Laplacian. Journal of Evolution Equations, 2003, 3, pp.443-461. 10.1007/978-3-0348-7924-8_24. hal-00115548

\section{HAL Id: hal-00115548 \\ https://hal.science/hal-00115548}

Submitted on 21 Nov 2006

HAL is a multi-disciplinary open access archive for the deposit and dissemination of scientific research documents, whether they are published or not. The documents may come from teaching and research institutions in France or abroad, or from public or private research centers.
L'archive ouverte pluridisciplinaire HAL, est destinée au dépôt et à la diffusion de documents scientifiques de niveau recherche, publiés ou non, émanant des établissements d'enseignement et de recherche français ou étrangers, des laboratoires publics ou privés. 


\title{
Minimization problems for eigenvalues of the Laplacian *
}

\author{
Antoine HENROT \\ Ecole des Mines and Institut Elie Cartan Nancy, \\ UMR 7502 CNRS and projet CORIDA, INRIA, \\ B.P. 239, 54506 Vandœuvre-lès-Nancy, France. \\ E-mail : henrot@iecn.u-nancy.fr
}

\begin{abstract}
This paper is a survey on classical results and open questions about minimization problems concerning the lower eigenvalues of the Laplace operator. After recalling classical isoperimetric inequalities for the two first eigenvalues, we present recent advances on this topic. In particular, we study the minimization of the second eigenvalue among plane convex domains. We also discuss the minimization of the third eigenvalue. We prove existence of a minimizer. For others eigenvalues, we just give some conjectures. We also consider the case of Neumann, Robin and Stekloff boundary conditions together with various functions of the eigenvalues.
\end{abstract}

AMS classification : 49Q10, 35P15, 49J20.

keywords : eigenvalues, minimization, isoperimetric inequalities, optimal domain

\section{Introduction}

Problems linking the shape of a domain to the sequence of its eigenvalues, or some of them, are among the most fascinating of mathematical analysis or differential geometry. In particular, problems of minimization of eigenvalues, or combination of eigenvalues, brought about many deep works since the early part of the twentieth century. Actually, this question appeared in the famous book of Lord Rayleigh "The theory of sound" ( for example in the edition of 1894). Thanks to some explicit computations and "physical evidence", Lord Rayleigh conjectured that the disk should minimize the first Dirichlet eigenvalue $\lambda_{1}$ of the Laplacian among every open sets of given measure.

It was indeed in the 1920's that Faber [21] and Krahn [34] solved simultaneously the Rayleigh's conjecture using a rearrangement technique. This classical proof is given at Theorem 1 of section 3 which is devoted to the first Dirichlet eigenvalue. We will also discuss the case of a multiconnected domain and present some open problems involving

\footnotetext{
*A shorter version of this paper appeared in Journal of Evolution Equations, volume 3 (2003), pp. 443-461 special issue dedicated to Philippe Bénilan
} 
the first eigenvalue. For other problems and a complete bibliography, we refer to [2], [41], [42], [50], [59], [60]; this kind of question is often called "isoperimetric inequalities for eigenvalues" in standard works, see also [6], [38] [48], [49]. In section 4, we investigate similar questions for the second eigenvalue. The open set, of given measure, which minimizes $\lambda_{2}$ is the union of two identical balls. This result is generally attributed to P. Szegö, as quoted by G. Pólya in [47]), but it was already contained (more or less explicitly) in one of the Krahn's papers, see [35]. In this section, we will also present very recent results about the minimization of $\lambda_{2}$ among convex plane domains. In section
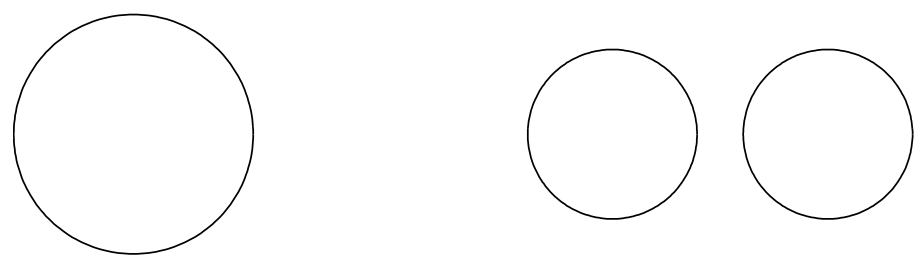

Figure 1: The ball minimizes $\lambda_{1}$ (left); the union of two identical balls minimizes $\lambda_{2}$ (right).

5, we look at the remaining eigenvalues of the Dirichlet-Laplacian. Actually a very few things are known! We only know the existence of an optimal domain for $\lambda_{3}$ and the fact that this domain is connected in dimension 2 and 3 . In section 6 , we will consider the so-called "Payne-Pólya-Weinberger" conjecture, solved by Ashbaugh and Benguria in the 90 's, concerning the ratio of the two first eigenvalues $\frac{\lambda_{2}}{\lambda_{1}}$. We will also present some open problems on other ratios. Finally, in section 7 , we will present some results about other boundary conditions: Neumann, Robin and also the Stekloff problem. We have decided here to restrict ourselves to the Laplacian operator on open (Euclidean) sets. Now, there are also beautiful results and conjectures e.g. for the bi-Laplacian $\Delta(\Delta)$. A good overview is given by B. Kawohl in the book [32], see also [37] and [5]. There are also many similar results on manifolds, see e.g. [39] or [60].

\section{Notations and prerequisites}

For the basic facts we recall here, we refer to any textbook on partial differential equations. For example, [17] or [20] are good standard references. Let $\Omega$ be a bounded open set in $\mathbb{R}^{N}$. In the case of Dirichlet boundary conditions, the convenient functional space is the Sobolev space $H_{0}^{1}(\Omega)$ which is defined as the closure of $C^{\infty}$ functions compactly supported in $\Omega$ for the norm $\|u\|_{H^{1}}:=\left(\int_{\Omega} u(x)^{2} d x+\int_{\Omega}|\nabla u(x)|^{2} d x\right)^{1 / 2}$. The Laplacian on $\Omega$ with Dirichlet boundary conditions is a self-adjoint operator with compact inverse, so there exists a sequence of positive eigenvalues (going to $+\infty$ ) and a sequence of corresponding eigenfunctions that we will denote respectively $0<\lambda_{1}(\Omega) \leq \lambda_{2}(\Omega) \leq \lambda_{3}(\Omega) \leq \ldots$ and 
$u_{1}, u_{2}, u_{3}, \ldots$. In other words, we have:

$$
\left\{\begin{array}{cc}
-\Delta u_{k}=\lambda_{k}(\Omega) u_{k} & \text { in } \Omega \\
u_{k}=0 & \text { on } \partial \Omega
\end{array}\right.
$$

We decide to normalize the eigenfunctions by the condition

$$
\int_{\Omega} u_{k}(x)^{2} d x=1 .
$$

The sequence of eigenfunctions defines an Hilbert basis of $L^{2}(\Omega)$. By hypo-analyticity of the Laplacian, each eigenfunction is analytic inside $\Omega$, its behavior on the boundary is governed by classical regularity results for elliptic partial differential equations. From the maximum principle and the Krein-Rutman Theorem, it follows that the first eigenfunction $u_{1}$ is non negative in $\Omega$ and positive as soon as $\Omega$ is connected. In particular, since $u_{2}$ is orthogonal to $u_{1}$, it has to change the sign in $\Omega$. The sets

$$
\Omega_{+}=\left\{x \in \Omega, u_{2}(x)>0\right\} \quad \text { and } \quad \Omega_{-}=\left\{x \in \Omega, u_{2}(x)<0\right\}
$$

are called the nodal domains of $u_{2}$. According to the Courant-Hilbert Theorem, these two nodal domains are connected subsets of $\Omega$. The set

$$
\mathcal{N}=\left\{x \in \Omega, u_{2}(x)=0\right\}
$$

is called the nodal line of $u_{2}$. When $\Omega$ is a plane convex domain, this nodal line hits the boundary of $\Omega$ at exactly two points, see Melas [36], or Alessandrini [1]. For general simply connected plane domains $\Omega$, it is still a conjecture, named after Larry Payne, the "Payne conjecture". We will also use the classical variational characterization of eigenvalues (Poincaré principle):

$$
\lambda_{k}(\Omega)=\min _{\begin{array}{c}
E_{k} \subset H_{0}^{1}(\Omega), \\
\text { subspace of } \operatorname{dim} k
\end{array}} \max _{v \in E_{k}, v \neq 0} \frac{\int_{\Omega}|\nabla v(x)|^{2} d x}{\int_{\Omega} v(x)^{2} d x} .
$$

This principle implies the following monotonicity for inclusion:

$$
\Omega_{1} \subset \Omega_{2} \Longrightarrow \lambda_{k}\left(\Omega_{1}\right) \geq \lambda_{k}\left(\Omega_{2}\right) .
$$

For the first eigenvalue, it reads

$$
\lambda_{1}(\Omega)=\inf _{v \in H_{0}^{1}(\Omega), v \neq 0} \frac{\int_{\Omega}|\nabla v(x)|^{2} d x}{\int_{\Omega} v(x)^{2} d x}
$$

the above infimum being achieved by the first eigenfunction. At last, the eigenvalues have a simple behavior with respect to homothety: if $t \Omega$ denotes the image of $\Omega$ by an homothety with ratio $t$, the eigenvalues of $t \Omega$ satisfy:

$$
\lambda_{k}(t \Omega)=\frac{\lambda_{k}(\Omega)}{t^{2}} .
$$


As a consequence, in two-dimensions, looking for the minimizer of $\lambda_{k}(\Omega)$ with a volume constraint is equivalent to look for a minimizer of the product $|\Omega| \lambda_{k}(\Omega)$.

In the sequel, we are interested in minimization problems like

$$
\min \left\{\lambda_{k}(\Omega), \Omega \text { open subset of } \mathbb{R}^{N},|\Omega|=A\right\}
$$

(where $|\Omega|$ denotes the measure of $\Omega$ and $A$ is a given constant). Sometimes, we will also consider other geometric or topologic constraints, this will be specified below.

\section{The first eigenvalue of the Dirichlet-Laplacian}

\subsection{The Rayleigh-Faber-Krahn inequality}

For the first eigenvalue, the basic result is (as conjectured by Lord Rayleigh):

Theorem 1 (Rayleigh-Faber-Krahn) Let $\Omega$ be any bounded open set in $\mathbb{R}^{N}$, let us denote by $\lambda_{1}(\Omega)$ its first eigenvalue for the Laplace operator with Dirichlet boundary conditions. Let $B$ be the ball of the same volume as $\Omega$, then

$$
\lambda_{1}(B)=\min \left\{\lambda_{1}(\Omega), \Omega \text { open subset of } \mathbb{R}^{N},|\Omega|=|B|\right\} .
$$

Proof : The classical proof makes use of the Schwarz spherical decreasing rearrangement. For every bounded open set $\omega$, let $\omega^{*}$ denotes the ball (centered at the origin) with the same volume as $\omega$. If $u$ is a non negative function in $\Omega$ which vanishes on $\partial \Omega$, its spherical decreasing rearrangement is defined as the function $u^{*}$ on $B=\Omega^{*}$ which has the following level sets:

$$
\forall c>0, \quad\left\{x \in B, u^{*}(x)>c\right\}=\{x \in \Omega, u(x)>c\}^{*} .
$$

In other words, the level sets of $u^{*}$ are the balls that we obtain by rearranging the sets of same level of $u$. We have a first easy consequence (by equi-measurability of the functions $u$ and $\left.u^{*}\right)$ :

$$
\int_{B} u^{*}(x)^{2} d x=\int_{\Omega} u(x)^{2} d x
$$

The following inequality involving the Dirichlet integrals of $u$ and $u^{*}$ is much harder to prove, but it is one of the main interest of the rearrangement techniques, we refer to [48] or [6] for the proof:

$$
\int_{B}\left|\nabla u^{*}(x)\right|^{2} d x \leq \int_{\Omega}|\nabla u(x)|^{2} d x .
$$

We can now apply (5) and (6) by choosing for the function $u$ the first eigenfunction $u_{1}$ of $\Omega$, it comes:

$$
\lambda_{1}(\Omega)=\frac{\int_{\Omega}\left|\nabla u_{1}(x)\right|^{2} d x}{\int_{\Omega} u_{1}(x)^{2} d x} \geq \frac{\int_{B}\left|\nabla u_{1}^{*}(x)\right|^{2} d x}{\int_{B} u_{1}^{*}(x)^{2} d x} \geq \lambda_{1}(B)
$$

the last inequality coming from (4), which yields the desired result. 


\subsection{The case of polygons}

We can ask the same question for the class of polygons with a given number $n$ of sides. Actually, the result is known only for $n=3$ and $n=4$ :

Theorem 2 (Pólya) The equilateral triangle has the least first eigenvalue among all triangles of given area. The square has the least first eigenvalue among all quadrilaterals of given area.

The proof relies on the same technique as the Rayleigh-Faber-Krahn Theorem with the difference that is now used the so-called Steiner symmetrization (see e.g. [48] or [31]). This symmetrization is performed with respect to an hyperplane $H$ : we transform a given set $\omega$ in a set $\omega^{*}$ symmetric w.r.t $H$ by moving the center of each segment of $\omega$ orthogonal to $H$ on $H$. Doing the same for the level set of a function allows to define the Steiner symmetrization of a given function. This symmetrization has the same properties (5) and (6) as the Schwarz rearrangement, therefore any Steiner symmetrization decreases the first eigenvalue.

By a sequence of Steiner symmetrization with respect to the mediator of each side, a given triangle converges to an equilateral one. We can do the same for a quadrilateral by alterning symmetrization w.r.t. mediator of sides and diagonals. It will be transformed into a square with the means of an infinite sequence of Steiner symmetrization. This is the idea of Pólya's proof.

Unfortunately, for $n \geq 5$ (pentagons and others), the Steiner symmetrization increases, in general, the number of sides. This prevents us to use the same technique. So a beautiful (and hard) challenge is to solve the

Open problem 1 Prove that the regular $n$-gone has the least first eigenvalue among all the $n$-gone of given area for $n \geq 5$.

This conjecture is supported by the classical isoperimetric inequality linking area and length for regular $n$-gones, see e.g. Theorem 5.1 in Osserman, [38]. Another kind of result that can be proved on polygons has been stated by J. Hersch in [28]:

Among all parallelograms with given distances between their opposite sides, the rectangle maximizes $\lambda_{1}$.

\subsection{Domains in a box}

Instead of looking at open sets just with a given volume, we could consider open sets constrained to lie into a given box $D$ (and also with a given volume). In other words, we could look for the solution of

$$
\min \left\{\lambda_{1}(\Omega), \Omega \subset D,|\Omega|=A \text { (given) }\right\} .
$$

According to the Theorem 7 of Buttazzo-DalMaso which will be stated below, the problem (7) has always a solution. Of course, if the constant $A$ is small enough in such a way 
that the ball of volume $A$ lies in the box $D$, it will provide the solution. Therefore, the interesting case is when the ball of volume $A$ is "too big" to stay into $D$. In this case, we can prove, at least formally, that the optimal domain, say $\Omega^{*}$ has to touch the boundary of $D$. Indeed, if it was not the case, and assuming $\Omega^{*}$ to be regular, we can use classical Hadamard's formula for variations of eigenvalues, see e.g. [52], [53] to get an optimality condition. This formula is the following: if we deform the domain $\Omega^{*}$ thanks to a deformation field $V$ such that if we set

$$
\Omega_{t}=(I d+t V)\left(\Omega^{*}\right)
$$

then the differential quotients

$$
\frac{\lambda_{1}\left(\Omega_{t}\right)-\lambda_{1}\left(\Omega^{*}\right)}{t}
$$

have a limit when $t$ goes to 0 . Moreover, this limit is given by the formula:

$$
d \lambda_{1}\left(\Omega^{*}, V\right)=-\int_{\partial \Omega^{*}}\left(\frac{\partial u_{1}}{\partial n}\right)^{2} V \cdot n d \sigma .
$$

where $\frac{\partial u_{1}}{\partial n}$ denotes the normal derivative of the eigenfunction $u_{1}$ and $V . n$ is the normal displacement of the boundary induced by the deformation field $V$. We have a similar formula for the first variation of the volume $\mathrm{Vol}$ :

$$
d V \operatorname{Vol}\left(\Omega^{*}, V\right)=\int_{\partial \Omega^{*}} \operatorname{V.nd\sigma } .
$$

Therefore, the optimal domain must satisfy a Lagrange identity like

$$
d \lambda_{1}\left(\Omega^{*}, V\right)=-c^{2} d V o l\left(\Omega^{*}, V\right)
$$

for every deformation field $V$ (with $c^{2}$ a Lagrange multiplier), which yields the following relation for the normal derivative of the first eigenfunction:

$$
\frac{\partial u_{1}}{\partial n}=c .
$$

Now, this relation (10) together with the p.d.e. (1) yields a well-known overdetermined problem whose only solution, according to J. Serrin cf [51], is a ball! Therefore, the optimal domain must touch $\partial D$. More precisely, the boundary of $\Omega^{*}$ has two kind of components:

- free components included in $D$,

- components lying on the boundary of $D$.

A natural question is to ask whether the free components are composed of pieces of spheres. We proved in a recent paper, see [24] that it is not the case: 


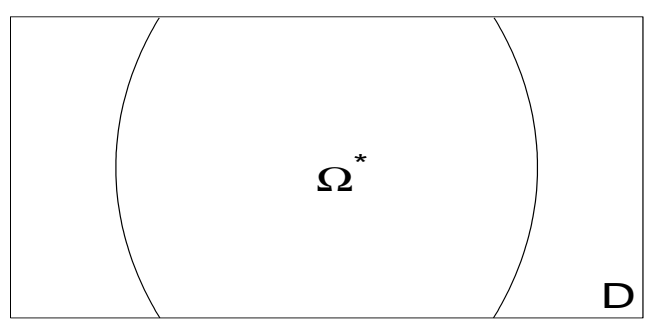

Figure 2: $\Omega^{*}$ solves the problem (7): the free components of $\partial \Omega^{*}$ are not arc of circles.

Proposition 2.1 The free components of the domain $\Omega^{*}$ which solve problem (7) cannot be pieces of spheres unless the ball of volume $A$ is the solution.

Proof : Let us assume that $\partial \Omega^{*}$ contains a piece of sphere $\gamma$. On $\gamma, \Omega^{*}$ satisfies the optimality condition (10). We put the origin at the center of the corresponding ball and we introduce the functions

$$
w_{i, j}(x, y)=x_{i} \frac{\partial u}{\partial x_{j}}-x_{j} \frac{\partial u}{\partial x_{i}}
$$

Then, we easily verify that

$$
\begin{gathered}
-\Delta w_{i, j}=\lambda_{1} w_{i, j} \text { in } \Omega^{*} \\
w_{i, j}=0 \text { in } \gamma \\
\frac{\partial w_{i, j}}{\partial n}=0 \text { in } \gamma .
\end{gathered}
$$

Now we conclude, using Hölmgren uniqueness theorem, that $w_{i, j}$ must vanish in a neighborhood of $\gamma$, so in the whole domain by analyticity. Now, if all these functions $w_{i, j}$ are identically 0 in $\Omega^{*}$, this would imply that $u$ is radially symmetric in $\Omega^{*}$ and therefore that $\Omega^{*}$ is a ball.

Nevertheless, there are some interesting open questions for this very simple minimization problem. For example:

Open problem 2 Let $\Omega^{*}$ be a solution of the minimization problem (7). Prove that the free components of the boundary of $\Omega^{*}$ are $C^{\infty}$ (or analytic). If $D$ is convex, is it true that $\Omega^{*}$ is convex?

\subsection{Multi-connected domains}

This section could also be entitled "How to place an obstacle" (see [23]). Let us consider a multi-connected domain $\Omega$ with one or several holes whose boundaries are denoted by $\Gamma_{0}, \Gamma_{1}, \ldots$, the outer boundary of $\Omega$ being denoted by $\Gamma$. We can consider many problems, letting the boundary conditions varying on the outer boundary and/or the holes. Let me 
mention below the results known by the author on such minimization and maximization problems.

- One hole, Dirichlet boundary condition on $\Gamma$ and $\Gamma_{0}$. J. Hersch in [27] proves: Of all such plane domains, with given area $A$, and given length $L$ and $L_{0}$ of its outer and inner boundary satisfying $L^{2}-L_{0}^{2}=4 \pi A$, the annular domain (two concentric circles) maximizes $\lambda_{1}$.

This result implies, in particular, that for a domain $\Omega$ of the kind $\Omega=B_{1} \backslash B_{0}$ (difference of two disks of given radii), $\lambda_{1}$ is maximal when the disks are concentric. This particular result has been rediscovered later and extended to the $N$-dimensional case by several authors: M. Ashbaugh and T. Chatelain in 1997 (private communication), E. Harrel, P. Kröger and K. Kurata in [23], Kesavan, see [33]. They also proved that $\lambda_{1}\left(B_{1} \backslash B_{0}\right)$ is a minimum when $B_{0}$ touches the boundary of $B_{1}$.

Open problem 3 Let $\Omega$ be a fixed domain and $B_{0}$ a ball of fixed radius. Prove that $\lambda_{1}\left(\Omega \backslash B_{0}\right)$ is minimal when $B_{0}$ touches the boundary of $\Omega$ (where?) and is maximum when $B_{0}$ is centered at a particular point of $\Omega$ (at what point?). In [23], one can find some interesting partial answers assuming convexity and/or symmetry properties for $\Omega$. They also give many illustrative examples. Actually, I think that the optimal center of $B_{0}$ depends on the radius and is not fixed (apart in the case of symmetries). When the radius of $B_{0}$ goes to zero, classical asymptotic formulae for eigenvalues of domains with small holes, see e.g. the review paper [22], lead one to think that the ball must be located at the maximal point of the first eigenvalue of the domain without holes. Of course, we can state the same question with a non circular hole of given measure: in such a case, we have to find not only the location but also the shape of the hole in order to minimize or maximize the first eigenvalue.
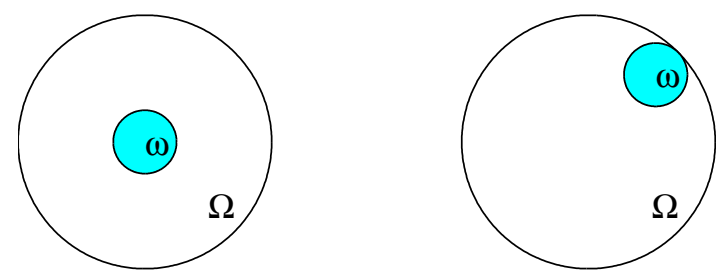

Figure 3: Position of the hole which maximizes $\lambda_{1}(\Omega \backslash \omega)$ (left); one position which minimizes $\lambda_{1}(\Omega \backslash \omega)$ (right).

- Several holes, Dirichlet boundary condition on the outer boundary $\Gamma$ and Neumann boundary condition on the boundary of the holes. L. Payne and H. Weinberger proves in [46]:

Among all multi-connected plane domains, with given area $A$, and given length $L$ 
of its outer boundary, the annular domain (two concentric circles) maximizes the first eigenvalue $\lambda_{1}$ with Dirichlet boundary condition on the outer boundary $\Gamma$ and Neumann boundary condition on the boundary of the holes.

- Several holes, Neumann boundary condition on the outer boundary $\Gamma$, Dirichlet boundary condition on one hole and Neumann boundary condition on the boundary of the other holes. J. Hersch proves in [27]:

Among all multi-connected plane domains, with given area $A$, and given length $L_{0}$ of the first inner boundary, the annular domain (two concentric circles) maximizes the first eigenvalue $\lambda_{1}$ with Neumann boundary condition on the outer boundary $\Gamma$, Dirichlet boundary condition on one hole and Neumann boundary condition on the boundary of the other holes.

\section{The second eigenvalue of the Dirichlet-Laplacian}

For the second eigenvalue, the minimizer is not one ball, but two!

Theorem 3 (Krahn-Szegö) The minimum of $\lambda_{2}(\Omega)$ among bounded open sets of $\mathbb{R}^{N}$ with given volume is achieved by the union of two identical balls.

Proof : Let $\Omega$ be any bounded open set, and let us denote by $\Omega_{+}$and $\Omega_{-}$its nodal domains. Since $u_{2}$ satisfies

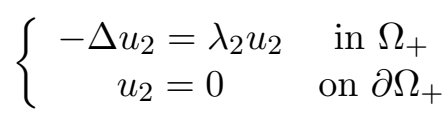

$\lambda_{2}(\Omega)$ is an eigenvalue for $\Omega_{+}$. But, since $u_{2}$ is positive in $\Omega_{+}$, it is the first eigenvalue (and similarly for $\Omega_{-}$):

$$
\lambda_{1}\left(\Omega_{+}\right)=\lambda_{1}\left(\Omega_{-}\right)=\lambda_{2}(\Omega) .
$$

We now introduce $\Omega_{+}^{*}$ and $\Omega_{-}^{*}$ the balls of same volume as $\Omega_{+}$and $\Omega_{-}$respectively. According to the Rayleigh-Faber-Krahn inequality

$$
\lambda_{1}\left(\Omega_{+}^{*}\right) \leq \lambda_{1}\left(\Omega_{+}\right), \quad \lambda_{1}\left(\Omega_{-}^{*}\right) \leq \lambda_{1}\left(\Omega_{-}\right) .
$$

Let us introduce a new open set $\tilde{\Omega}$ defined as

$$
\tilde{\Omega}=\Omega_{+}^{*} \cup \Omega_{-}^{*} .
$$

Since $\tilde{\Omega}$ is disconnected, we obtain its eigenvalues by gathering and reordering the eigenvalues of $\Omega_{+}^{*}$ and $\Omega_{-}^{*}$. Therefore,

$$
\lambda_{2}(\tilde{\Omega}) \leq \max \left(\lambda_{1}\left(\Omega_{+}^{*}\right), \lambda_{1}\left(\Omega_{-}^{*}\right)\right) .
$$

According to (11), (12) we have

$$
\lambda_{2}(\tilde{\Omega}) \leq \max \left(\lambda_{1}\left(\Omega_{+}\right), \lambda_{1}\left(\Omega_{-}\right)\right)=\lambda_{2}(\Omega) .
$$


This shows that the minimum of $\lambda_{2}$ is to be obtained among the union of balls. But, if the two balls would have different radii, we would decrease the second eigenvalue by shrinking the largest one and dilating the smaller one (without changing the total volume). Therefore, the minimum is achieved by the union of two identical balls.

Being disappointed that the minimizer be not a connected set (it's hard to hit with one hand on a non-connected drum!), we could be interested in solving the minimization problem for $\lambda_{2}$ among connected sets. Unfortunately, a connectedness constraint does not really change the situation. Indeed, let us consider the following domain (see Figure 4) $\Omega_{\varepsilon}$, obtained by joining the union of the two previous balls $\Omega$ by a thin pipe of width $\varepsilon$. We say that $\Omega_{\varepsilon} \gamma$-converges to $\Omega$ if the resolvent operators $T_{\varepsilon}$ associated with the
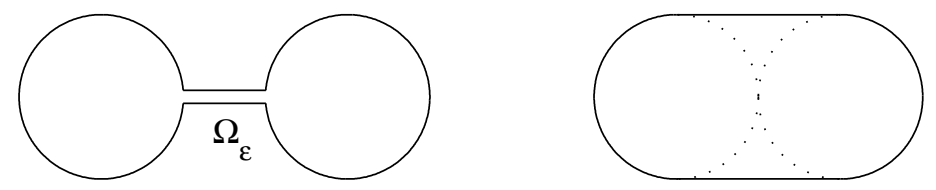

Figure 4: A minimizing sequence of connected domains (left), the stadium does not minimize $\lambda_{2}$ among convex sets of given volume (right)

Laplace-Dirichlet operator on $\Omega_{\varepsilon}$ simply converge to the corresponding operator $T$ on $\Omega$, see e.g. [18]. By a compactness argument, see [12], [26] it can be proved that this simple convergence implies the convergence in the operator norm and therefore the convergence of the eigenvalues. Now, it is easy to verify, see [11], [26], that in the above situation $\Omega_{\varepsilon}$ $\gamma$-converges to $\Omega$ what yields $\lambda_{2}\left(\Omega_{\varepsilon}\right) \rightarrow \lambda_{2}(\Omega)$ and therefore:

$$
\inf \left\{\lambda_{2}(\Omega), \Omega \subset \mathbb{R}^{N}, \Omega \text { connected },|\Omega|=c\right\}=\min \left\{\lambda_{2}(\Omega), \Omega \subset \mathbb{R}^{N},|\Omega|=c\right\}
$$

what shows that this infimum is not achieved (actually, we can prove that the union of two balls is the unique minimizer of $\lambda_{2}$ up to displacements and zero-capacity subsets).

Now, the problem becomes again interesting if we ask the question to find the convex domain, of given area, which minimizes $\lambda_{2}$. For sake of simplicity, we restrict us here to the two-dimensional case. Existence of a minimizer $\Omega^{*}$ is easy to obtain (see [15] and [24], [25]). In a paper of 1973 [55], Troesch did some numerical experiments which led him to conjecture that the solution was a stadium: the convex hull of two identical tangent disks. It is actually the convex domain which is the closest to the solution without convexity constraint. In [24], we refute this conjecture:

Theorem 4 (Henrot-Oudet) The stadium, convex hull of two identical tangent disks, does not realize the minimum of $\lambda_{2}$ among plane convex domains of given area.

Indeed, the proof is exactly the same as the proof of the above Proposition 2.1. Nevertheless, a more precise analysis and some numerical experiments show that the minimizer, say $\Omega^{*}$, is very close to the stadium. Actually, we prove in [24], [25]: 


\section{Theorem 5 (Henrot-Oudet)}

Regularity The minimizer $\Omega^{*}$ is at least $C^{1}$ and at most $C^{2}$.

Simplicity The second eigenvalue of $\Omega^{*}$ is simple.

Geometry The minimizer $\Omega^{*}$ has two (and only two) segments in its boundary and these segments are parallel.

Proof : Below, we just give the main lines of the proof, see [25] for the details. Of course our main ingredient in the following proof will be optimality conditions satisfied on the boundary of $\Omega^{*}$. We use again the classical tool of derivative with respect to the domain for the eigenvalues. The difficulty is to take care of the convexity constraint when deforming the original domain $\Omega^{*}$ by a vector field $V$. Indeed, if we perform a small deformation of a strictly convex part of the boundary of $\Omega^{*}$, this part will not remain necessarily convex, but we can use the fact that the difference between the deformed boundary ant its convex hull is so small, that for first order terms, the formulae of derivative still holds. On the contrary, for segments included in the boundary, it is no longer true. Therefore, we need to make a distinction between the strictly convex parts of the boundary and the segments included in the boundary. More precisely, we are still able to get an optimality condition of the kind (10): the normal derivative of the second eigenfunction must be constant on the strictly convex parts of the boundary of $\Omega^{*}$. On the segments, we get a weaker relation also involving the normal derivative.

Regularity - At least $C^{1}$ : we assume, for a contradiction, that $\Omega^{*}$ has two distinct supporting lines at some point $x_{0}$ of its boundary. Then, we prove that we can decrease the product $\left|\Omega^{*}\right| \lambda_{2}\left(\Omega^{*}\right)$ by cutting a small cap of size $\varepsilon$. The key point is to estimate precisely the second eigenvalue of the new domain. We use, for that purpose, the Poincaré variational characterization of the eigenvalues.

- At most $C^{2}$ : this point can be proved after the last point of the item "Geometry". If $\partial \Omega^{*}$ was more than $C^{2}$, by classical Schauder regularity results for elliptic p.d.e., the eigenfunction $u$ would be $C^{2}$ up to the boundary. We assume that the segments are parallel to the first axis of coordinates and we introduce the function $\frac{\partial u}{\partial x}$. Using Hopf's Lemma and the optimality condition on segments, we are able to prove that $\frac{\partial u}{\partial x}$ has at least four nodal lines starting on each segments. Closing these nodal lines, we define at least three nodal domains of $\frac{\partial u}{\partial x}$ strictly contained in $\Omega^{*}$. Now $\frac{\partial u}{\partial x}$ being an eigenfunction associated to $\lambda_{2}$, the Courant-Fischer nodal domain Theorem would lead to the fact that $\lambda_{2}$ is at least the third eigenvalue of a strict subdomain of $\Omega^{*}$, which is a contradiction with the monotonicity of eigenvalues.

Simplicity The proof is based on the following property: assume that $\Omega$ is a domain which has a multiple eigenvalue of order $m$ :

$$
\lambda_{k+1}(\Omega)=\lambda_{k+2}(\Omega)=\ldots=\lambda_{k+m}(\Omega) .
$$


Then, we can always find a deformation field $V \in C^{1,1}\left(\mathbb{R}^{N}, \mathbb{R}^{N}\right)$, preserving the volume and such that, if we set

$$
\Omega_{t}=(I d+t V)(\Omega)
$$

we have, for $t>0$ small enough

$$
\lambda_{k+1}\left(\Omega_{t}\right)<\lambda_{k+1}(\Omega)=\lambda_{k+m}(\Omega)<\lambda_{k+m}\left(\Omega_{t}\right) .
$$

The proof of this fact uses computations of the domain derivative for multiple eigenvalues. The previous result has the following consequence about minimization of eigenvalues: if $\Omega^{*}$ is a domain minimizing the $k$-th eigenvalue and if $\lambda_{k}\left(\Omega^{*}\right)$ is not simple, necessarily we have

$$
\lambda_{k-1}\left(\Omega^{*}\right)=\lambda_{k}\left(\Omega^{*}\right) .
$$

Actually, numerical experiments show that this relation holds in every case, see [40]: the domain which minimizes $\lambda_{k}(\Omega), k \geq 2$ (with a volume constraint but without convexity constraint) always satisfies (13). Coming back to a convex domain $\Omega^{*}$ minimizing $\lambda_{2}$, we know that $\lambda_{1}\left(\Omega^{*}\right)$ is simple and therefore (13) cannot hold.

Geometry - There is at least one segment on the boundary, otherwise the normal derivative of $u$ would be zero on the whole boundary, because it is constant (by optimality condition) and it has to be zero where the nodal line hits the boundary. It is easy to see that it is impossible, e.g. using one more time Hölmgren uniqueness argument.

- There are at least two segments on the boundary, otherwise the nodal line would have to close on the same segment and we could adapt an idea of Melas [36] to reach a contradiction.

- There are at most two segments on the boundary, otherwise we consider the segment $\mathcal{S}$ in the boundary which does not meet the nodal line. We put it horizontal and we introduce the auxiliary eigenfunction $v_{t}=t u+\frac{\partial u}{\partial x}$. Thanks to the optimality condition on segments, we prove that this function has at least three nodal lines starting on $\mathcal{S}$ for $t$ small. Then, we reach a contradiction by letting $t$ increasing up to a critical value where $v_{t}$ would have all its derivatives which vanish up to the second order at some point and therefore everywhere.

- The two segments have to be parallel. To see that, we use some Rellich-PucciSerrin formulae for a well-chosen vector field together with the optimality conditions. This allow us to prove that the angle between the two segments has to vanish.

Open problem 4 Prove that a plane convex domain $\Omega^{*}$ which minimizes $\lambda_{2}$ (among convex domains of given area) has two perpendicular axes of symmetry. 


\section{Other eigenvalues of the Dirichlet-Laplacian}

The minimization problem becomes much more complicated for the other eigenvalues! One of the only known result is the following, cf [10] and [58]:

Theorem 6 (Bucur-Henrot and Wolff-Keller) There exists a set $\Omega_{3}^{*}$ which minimizes $\lambda_{3}$ among the (quasi)-open sets of given volume. Moreover $\Omega_{3}^{*}$ is connected in dimension $N=2$ or 3 .

The question of identifying the optimal domain $\Omega_{3}^{*}$ remains open. The conjecture is the following:

Open problem 5 Prove that the optimal domain for $\lambda_{3}$ is a ball in dimension 2 and 3 and the union of three identical balls in dimension $N \geq 4$.

Wolff and Keller have proved in [58] that the disk is a local minimizer for $\lambda_{3}$. There are two key-points in the existence proof of the above theorem. The first one is a more general result of Buttazzo-Dal Maso (already cited earlier), see [12]:

Theorem 7 (Buttazzo-Dal Maso) Let $D$ be a fixed ball in $\mathbb{R}^{N}$. For every fixed integer $k \geq 1$ and $c$ fixed real number $0<c<|D|$ the problem

$$
\min \left\{\lambda_{k}(\Omega) ; \quad \Omega \subset D, \quad|\Omega|=c\right\}
$$

has a solution.

More generally, the existence result remains valid for any function $\Phi\left(\lambda_{1}, \ldots, \lambda_{k}\right)$ of the eigenvalues non decreasing in each of its arguments.

This theorem does not solve the general problem of existence of a minimizer for $\lambda_{k}(\Omega)$ since we assume to work with "confined" sets (that is to say, sets included in a box $D$ ). In order to remove this assumption in [10], we used a "concentration-compactness" argument together with the Wolff-Keller's result proving that the minimizer of $\lambda_{3}$ (if it exists) should be connected in dimension 2 and 3 (this is the second key-point). Here is the more general result they prove in [58]. Let us denote by $\Omega_{n}^{*}$ an open set which minimizes $\lambda_{n}$ (among open sets of volume 1) and $\lambda_{n}^{*}=\lambda_{n}\left(\Omega_{n}^{*}\right)$ the minimal value of $\lambda_{n}$. We will also denote by $t \Omega$ the image of $\Omega$ by an homothety of ratio $t$. Then, we have:

Theorem 8 (Wolf-Keller) Let us assume that $\Omega_{n}^{*}$ is the union of (at least) two disjoints sets, each of them with positive measure. Then

$$
\left(\lambda_{n}^{*}\right)^{N / 2}=\left(\lambda_{i}^{*}\right)^{N / 2}+\left(\lambda_{n-i}^{*}\right)^{N / 2}=\min _{1 \leq j \leq(n-1) / 2}\left(\left(\lambda_{j}^{*}\right)^{N / 2}+\left(\lambda_{n-j}^{*}\right)^{N / 2}\right)
$$

where, in the previous equality, $i$ is a value of $j \leq(n-1) / 2$ which minimizes the sum $\left(\lambda_{j}^{*}\right)^{N / 2}+\left(\lambda_{n-j}^{*}\right)^{N / 2} \cdot$ Moreover

$$
\Omega_{n}^{*}=\left[\left(\frac{\lambda_{i}^{*}}{\lambda_{n}^{*}}\right)^{1 / 2} \Omega_{i}^{*}\right] \bigcup\left[\left(\frac{\lambda_{n-i}^{*}}{\lambda_{n}^{*}}\right)^{1 / 2} \Omega_{n-i}^{*}\right] .
$$


We have seen that the value of $\lambda_{n}^{*}$ is not known unless for $n=1$ or 2 . Let us prove for example, that the optimal domain is connected in dimension 2 . Indeed, if it was not connected, according to Theorem 8 , we should have $\lambda_{3}^{*}=\lambda_{1}^{*}+\lambda_{2}^{*}(i=1$ is the only possible value here). Now $\lambda_{1}^{*}=\pi j_{0,1}^{2}=18.168$.. ( $j_{0,1}$ is the first zero of the Bessel function $J_{0}$ ) while, according to Theorem $3, \lambda_{2}^{*}=2 \lambda_{1}^{*}=36.336$. Therefore $\lambda_{1}^{*}+\lambda_{2}^{*}=54.504$. But since $\lambda_{3}^{*}$ is, by definition, lower or equal to the third eigenvalue of the unit disk $\lambda_{3}\left(D_{1}\right)=\pi j_{1,1}^{2}=46.125 .$. , we see that it cannot be equal to $\lambda_{1}^{*}+\lambda_{2}^{*}$.

The same kind of computation works in dimension 3 , but not in higher dimension. This is the reason why we think that the minimizer is the union of three identical balls in dimension greater than 4 . To prove that the disk is a local minimizer of $\lambda_{3}$, Wolff and Keller use some precise perturbation argument. More precisely, they show that the third eigenvalue of a domain $\Omega_{\varepsilon}$ given in polar coordinates by $r=R(\theta, \varepsilon)$ where $R$ has an expansion like

$$
R(\theta, \varepsilon)=1+\varepsilon \sum_{n=-\infty}^{\infty} a_{n} e^{i n \theta}+\varepsilon^{2} \sum_{n=-\infty}^{\infty} b_{n} e^{i n \theta}+O\left(\varepsilon^{3}\right)
$$

is given by

$$
\lambda_{3}\left(\Omega_{\varepsilon}\right)=\pi j_{1,1}^{2}\left(1+2|\varepsilon|\left|a_{2}\right|\right)+O\left(\varepsilon^{2}\right) .
$$

In the case where $a_{2} \neq 0$, we immediately get the result. When $a_{2}=0$ it is necessary to look at the following term in the expansion, but the conclusion is the same. For the fourth
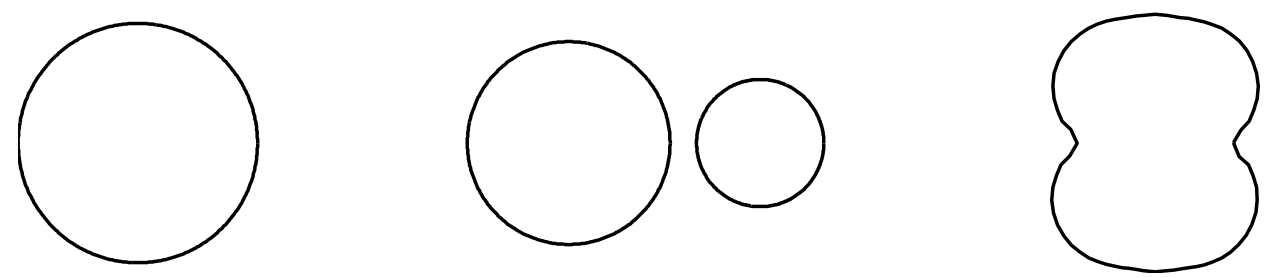

Figure 5: The disk probably minimizes $\lambda_{3}$ (left); two disks which probably minimize $\lambda_{4}$ (center); a domain candidate to minimize $\lambda_{5}$ (right).

eigenvalue, it is conjectured that the minimum is attained by the union of two balls whose radii are in the ratio $\sqrt{\frac{j_{0,1}}{j_{1,1}}}$ in dimension 2 , where $j_{0,1}$ et $j_{1,1}$ are respectively the two first zeros of the Bessel functions $J_{0}$ et $J_{1}$, cf Figure 5 , but it is not proved!

Open problem 6 Prove that the optimal domain for $\lambda_{4}$ is the union of two balls whose radii are in the ratio $\sqrt{\frac{j_{0,1}}{j_{1,1}}}$ in dimension 2 . 
Looking at the previous results and conjectures, P. Szegö asked the following question: Is it true that the minimizer of any eigenvalue of the Laplace-Dirichlet operator is a ball or a union of balls?

The answer to this question is NO. For example, Wolff and Keller remarked that the thirteenth (!) eigenvalue of a square is lower than the thirteenth eigenvalue of any union of disks of same area. Actually, it is not necessary to go to the 13th eigenvalue. Numerical experiments, cf [40] and Figure 5, show that for the $n$-th eigenvalue with $n$ larger or equal to 5 the minimizer is no longer a ball or a union of balls.

Let us state now some new open problems:

Open problem 7 Prove that there exist a minimizer for $\lambda_{n}$ among open sets of given volume. The technique we used in [10] allows us to prove such an existence result as soon as we are able to prove that the minimizers for $\lambda_{k}, k=1 \ldots n-1$ are indeed bounded.

Study the regularity and the geometric properties (e.g. symmetries) of such a minimizer.

\section{Optimizing functions of eigenvalues}

\subsection{Maximizing ratios of eigenvalues}

In 1955 L. Payne, G. Pólya and H. Weinberger in [43] considered the problem of bounding ratios of eigenvalues. In particular, they proved that the ratio $\frac{\lambda_{2}}{\lambda_{1}}$ is less than or equal to 3 (in dimension 2). They were led to conjecture that the optimal domain for this ratio is a disk. This conjecture has been proved 35 years later by M. Ashbaugh and R. Benguria, see [3] for the two-dimensional case and [4] for the $N$-dimensional case.

Theorem 9 (Ashbaugh-Benguria) The ball maximizes the ratio $\frac{\lambda_{2}}{\lambda_{1}}$.

Their (clever) proof uses

- a variational characterization for the difference $\lambda_{2}-\lambda_{1}$,

- the Brouwer fixed point theorem,

- a sharp use of some rearrangement inequalities,

- another inequality due to Chiti,

- a careful study of properties of Bessel functions.

For ratios of eigenvalues, many problems remain open. A good overview and discussion on previous results is given in [2]. Below, some of them are listed 
Open problem 8 (see [43]) Prove that the disk maximizes the quotient $\frac{\lambda_{2}+\lambda_{3}}{\lambda_{1}}$ among plane domains of given area.

Prove that the ball maximizes the quotient $\frac{\sum_{i=2}^{N+1} \lambda_{i}}{\lambda_{1}}$ among domains of $\mathbb{R}^{N}$ with given area.

Open problem 9 Prove existence of a domain which maximizes the following ratios, study the geometric properties of such maximizers, if possible identify it

- $\frac{\lambda_{3}}{\lambda_{1}}$ (in the plane this is not the disk)

- $\frac{\lambda_{N+2}}{\lambda_{1}}$ in $\mathbb{R}^{N}$ : it should be the ball

- $\frac{\lambda_{m+1}}{\lambda_{m}}$

- $\frac{\lambda_{2 m}}{\lambda_{m}}$

\subsection{Other functions of eigenvalues}

We have already mentioned in Theorem 7 that any function of the kind $\Omega \mapsto \Phi\left(\lambda_{1}(\Omega), \lambda_{2}(\Omega)\right)$ with $\Phi$ non decreasing with respect to each argument, admits a minimizer among (quasi)open sets of given volume. Note than none of the above ratios can be handled by this theorem. In an interesting paper [9], D. Bucur, G. Buttazzo and I.Figueiredo extended this result:

Theorem 10 (Bucur, Buttazzo, Figueiredo) Let $\Phi: \overline{\mathbb{R}}^{2} \rightarrow \mathbb{R}$ be a lower semi-continuous function, $D$ a given box and $A$ a given constant. Then the problem

$$
\min \left\{\Phi\left(\lambda_{1}(\Omega), \lambda_{2}(\Omega)\right), \Omega \subset D,|\Omega| \leq A\right\}
$$

has always a solution.

A more precise statement could be: either an optimal domain exists or for the minimizing sequence $\Omega_{n}$ we have $\Phi\left(\lambda_{1}\left(\Omega_{n}\right), \lambda_{2}\left(\Omega_{n}\right)\right) \rightarrow-\infty$. In this last case, we can choose as a minimizer the empty set. This is the case, for example for the gap function $\Phi\left(\lambda_{1}, \lambda_{2}\right)=$ $\lambda_{1}-\lambda_{2}$. Indeed, if we consider a sequence of domains

$$
\Omega_{\epsilon}=B(0, \epsilon) \cup_{i=1}^{N_{\epsilon}} B\left(x_{i}, a_{\epsilon} \epsilon\right)
$$

where $a_{\epsilon}<1$ and $N_{\epsilon}$ are chosen such that $\left|\Omega_{\epsilon}\right|=A$ and $\lambda_{i}\left(\Omega_{\epsilon}\right)=\lambda_{i}(B(0, \epsilon))$ for $i=1,2$, then

$$
\lambda_{1}\left(\Omega_{\epsilon}\right)-\lambda_{2}\left(\Omega_{\epsilon}\right) \rightarrow-\infty .
$$

The main ingredient of the proof of this theorem is the closedness (in the plane) of the set 


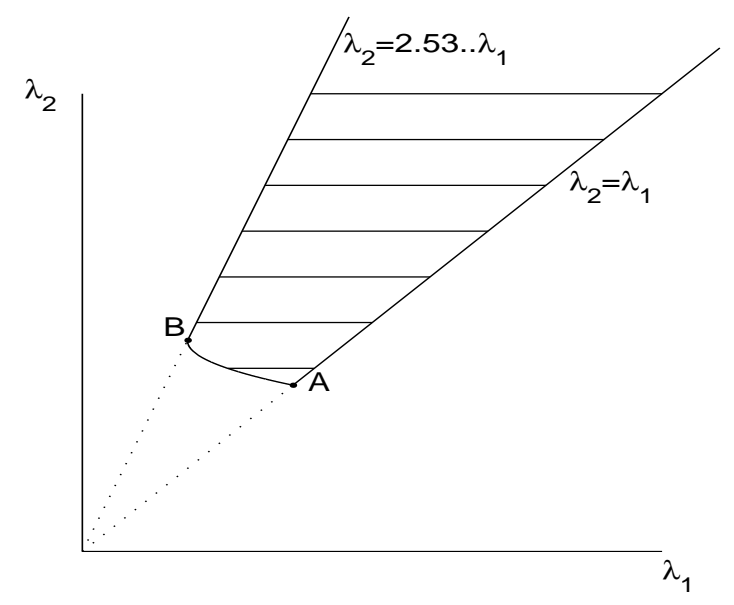

Figure 6: Range, in the plane $\left(\lambda_{1}, \lambda_{2}\right)$, of the possible values for the two first eigenvalues of a domain of given area.

$\mathcal{E}=\left\{\left(\lambda_{1}(\Omega), \lambda_{2}(\Omega)\right), \Omega \subset D,|\Omega| \leq A\right\}$. This set is represented in Figure 6. It is of course above the first bisectrix and, according to Ashbaugh-Benguria Theorem 9, it is below the line $y=2.5387 x$ ( 2.5387 is the value of the quotient for the disk). The point $A$ in the Figure corresponds to the two identical balls (Krahn-Szegö Theorem 3) while the point $B$ corresponds to one ball (Rayleigh-Faber-Krahn). Indeed, they proved that this set $\mathcal{E}$ is convex in the $x$ and the $y$ direction.

Open problem 10 Prove that the set $\mathcal{E}$ defined above is convex.

Among various combinations of the two first eigenvalues, we can also consider for example $\lambda_{1}+\lambda_{2}$ : what is the set which minimizes the sum of the two first eigenvalues? It is not the disk, since it does not satisfy the generalized optimality conditions (see e.g. [14], [13]). More generally, we can ask this question for any convex combination of the two first eigenvalues of the kind $t \lambda_{1}+(1-t) \lambda_{2}$. This question has a simple geometric interpretation: the wanted minimizer is indeed the first point of $\mathcal{E}$ we reach when making a line of equation $t x+(1-t) y=c$ approach the set $\mathcal{E}$ (by increasing $c$ ). In particular, for $t=1$ the solution is a ball while for $t=0$ it is given by two balls.

Open problem 11 For what value of $t$, the set which minimizes $t \lambda_{1}+(1-t) \lambda_{2}$ is no longer convex, no longer connected (perhaps $t=1$ for that second question)? 


\section{Eigenvalues of the Laplacian with other boundary condi- tions}

\subsection{Neumann boundary conditions}

The eigenvalues of the Laplacian with Neumann boundary conditions are also called the eigenvalues of the free membrane (in the case of Dirichlet boundary conditions, we speak about the fixed membrane). We will denote it by $0=\mu_{1}(\Omega) \leq \mu_{2}(\Omega) \leq \mu_{3}(\Omega) \leq \ldots$ (the first eigenvalue is zero, corresponding to constant functions). They solve

$$
\left\{\begin{array}{cc}
-\Delta u_{k}=\mu_{k}(\Omega) u_{k} & \text { in } \Omega \\
\frac{\partial u_{k}}{\partial n}=0 & \text { on } \partial \Omega .
\end{array}\right.
$$

Minimizing the eigenvalues of the Laplacian with Neumann boundary conditions, with a volume constraint, is a trivial problem. Indeed, if we consider a long thin rectangle like ] $0, L[\times] 0, l\left[\right.$, its $n$-th eigenvalue will be (for $L$ large enough) $\mu_{n}=\frac{(n-1)^{2} \pi^{2}}{L^{2}}$. Therefore, letting $L \rightarrow+\infty$, we see that

$$
\inf \left\{\mu_{n}(\Omega),|\Omega|=A\right\}=0 .
$$

Moreover, the infimum is attained for any open set which has at least $n$ connected components. This shows that limiting the diameter of $\Omega$ does not improve the interest of the question! Now, if we assume that the domains must be convex and with a given diameter, then the infimum is not zero, but it is not achieved! Actually, L. Payne and H. Weinberger proved in [45] the following inequality for convex domains $\Omega$ in $\mathbb{R}^{N}$ with given diameter $d$ :

$$
\mu_{2}(\Omega) \geq\left(\frac{\pi}{d}\right)^{2}
$$

This lower bound is optimal but not attained: any domain shrinking to a one-dimensional segment $[0, d]$ has its second eigenvalue which converges to the lower bound.

If we want to get a really interesting problem for eigenvalues of the Laplacian with Neumann boundary conditions, we must consider the problem of the maximization instead of the minimization:

Theorem 11 (Szegö,Weinberger) The ball maximizes the second Neumann eigenvalue among open sets of given volume.

In the two-dimensional case, the proof (using conformal maps) was given by G. Szegö in [54]. It has been generalized to any dimension by H. Weinberger in [56]. We must also mention that Szegö and Weinberger in the above-mentioned papers have proved in two-dimensions that

$$
\frac{1}{\mu_{2}}+\frac{1}{\mu_{3}} \text { is minimal for the disk. }
$$

Of course this result implies Theorem 11 since the second eigenvalue of the disk is double. Now, in higher dimensions, it is still an open problem: 
Open problem 12 Prove that

$$
\sum_{i=2}^{N+1} \frac{1}{\mu_{i}(\Omega)}
$$

is minimal for the ball among all domains with a given volume.

More generally, the existence of a convex domain which maximizes the $n$-th Neumann eigenvalue $\mu_{n}$ (with given volume) has been proved in [16]. So, we are also led to the following open problem(s):

Open problem 13 Prove that there exists an open set (of given volume) which maximizes the $n$-th Neumann eigenvalue $\mu_{n}$, for $n \geq 3$. If possible, identify this maximizer.

\subsection{Robin boundary condition}

The eigenvalues of the Laplacian with Robin boundary conditions are called the eigenvalues of the elastically supported membrane. We will denote them by $0<\nu_{1}(\alpha, \Omega) \leq \nu_{2}(\alpha, \Omega) \leq$ $\nu_{3}(\alpha, \Omega) \leq \ldots$ where $\alpha$ is a parameter, $0<\alpha<1$ (the cases $\alpha=0$ or 1 obviously correspond to Neumann or Dirichlet conditions). The p.d.e. system is

$$
\left\{\begin{array}{cc}
-\Delta u_{k}=\nu_{k}(\alpha, \Omega) u_{k} & \text { in } \Omega \\
\alpha u+(1-\alpha) \frac{\partial u_{k}}{\partial n}=0 & \text { on } \partial \Omega .
\end{array}\right.
$$

In two dimensions, we recover the Rayleigh-Faber-Krahn inequality; this result is not well known, it is due to M.H. Bossel in her thesis:

Theorem 12 (Bossel) The disk minimizes the first eigenvalue of the Robin problem among open sets with a given volume (for every value of $\alpha \in] 0,1]$ ).

Her proof uses a new variational method, see [7]. This method is inspired by that of extremal length.

Open problem 14 Generalize Bossel's Theorem to dimension $N$.

Open problem 15 (see [44]) For what values of $\alpha$, the ratio $\frac{\lambda_{2}}{\lambda_{1}}$ achieves its maximum for the disk? Let us remark that Problem 14 is already stated in Daners, see [19] where a lower bound for $\nu_{1}$ is given.

\subsection{Stekloff eigenvalue problem}

The Stekloff eigenvalue problem is the following:

$$
\left\{\begin{array}{cc}
\Delta u=0 & \text { in } \Omega \\
\frac{\partial u}{\partial n}=p u & \text { on } \partial \Omega
\end{array} .\right.
$$


We will denote its eigenvalues by $0=p_{1}(\Omega) \leq p_{2}(\Omega) \leq p_{3}(\Omega) \leq \ldots$ (the first eigenvalue is zero, corresponding to constant functions). Like in the Neumann case, it is the problem of maximization of the eigenvalues which is interesting here.

Theorem 13 (Weinstock,Brock) The ball maximizes the second Stekloff eigenvalue among open sets of given volume.

R. Weinstock gave the proof of this theorem in the two-dimensional case in [56]. His proof was inspired by the one of Szegö for the free membrane problem. F. Brock in [8] proved actually a sharper inequality, namely:

Let $\Omega$ be a bounded domain in $\mathbb{R}^{N}$ and $R$ the radius of the ball $\Omega^{*}$ of same volume than $\Omega$, then

$$
\sum_{i=2}^{N+1} \frac{1}{p_{i}(\Omega)} \geq N R
$$

the equality sign in (21) is attained if $\Omega$ is a ball. It is clear that (21) implies the above theorem since $p_{2}\left(\Omega^{*}\right)=1 / R$ has multiplicity $N$ for the ball. I must also mention that J. Hersch and L. Payne have already proved (21) in two-dimensions in [29] and that they have also proved a sharper inequality, together with M.M. Schiffer in [30], namely: the disk maximizes the product $p_{2}(\Omega) p_{3}(\Omega)$ among plane open sets of given volume.

Open problem 16 Study the maximization problem for other Stekloff eigenvalues.

Open problem 17 Prove that the $N$-ball maximizes the product $\Pi_{k=2}^{N+1} p_{k}(\Omega)$ among open sets in $\mathbb{R}^{N}$ with given volume.

\section{References}

[1] G. Alessandrini, Nodal lines of eigenfunctions of the fixed membrane problem in general convex domains, Comment. Math. Helv., 69 (1994), no. 1, 142-154.

[2] M.S. Ashbaugh, Open problems on eigenvalues of the Laplacian, Analytic and Geometric Inequalities and Their Applications, T. M. Rassias and H. M. Srivastava (editors), vol. 4787, Kluwer 1999.

[3] M.S. Ashbaugh, R. Benguria, Proof of the Payne-Pólya-Weinberger conjecture, Bull. Amer. Math. Soc., 25 nº1 (1991), 19-29.

[4] M.S. Ashbaugh, R. Benguria, A sharp bound for the ratio of the first two eigenvalues of Dirichlet Laplacians and extensions, Ann. of Math. 135 (1992), no. 3, 601-628.

[5] M.S. Ashbaugh, R. Benguria, On Rayleigh's conjecture for the clamped plate and its generalization to three dimensions, Duke Math. J., 78 (1995), 1-17. 
[6] C. Bandle Isoperimetric inequalities and applications. Monographs and Studies in Mathematics, 7. Pitman, Boston, Mass.-London, 1980.

[7] M.H. Bossel, Membranes élastiquement liées: extension du théorème de RayleighFaber-Krahn et de l'inégalité de Cheeger, C. R. Acad. Sci. Paris Sr. I Math. 302 (1986), no. 1, 47-50.

[8] F. BRock, An isoperimetric inequality for eigenvalues of the Stekloff problem, Z. Angew. Math. Mech., 81 (2001), no. 1, 69-71.

[9] D. Bucur, G. Buttazzo, I. Figueiredo, On the attainable eigenvalues of the Laplace operator, SIAM J. Math. Anal., 30 (1999), no. 3, 527-536 .

[10] D. Bucur, A. Henrot, Minimization of the third eigenvalue of the Dirichlet Laplacian, Proc. Roy. Soc. London, 456 (2000), 985-996.

[11] D. Bucur, J.P. Zolesio, N-dimensional shape optimization under capacitary constraints, J. of Diff. Eq.,123 n 2 (1995), 504-522.

[12] G. Buttazzo, G. Dal Maso, An Existence Result for a Class of Shape Optimization Problems, Arch. Rational Mech. Anal., 122 (1993), 183-195.

[13] T. Chatelain, M. Choulli Clarke generalized gradient for eigenvalues, Commun. Appl. Anal., 1 (1997), no. 4, 443-454.

[14] S.J. Cox, The generalized gradient at a multiple eigenvalue, J. Funct. Anal., 133 (1995), no. 1, 30-40.

[15] S.J. Cox, M. Ross Extremal eigenvalue problems for starlike planar domains, J. Differential Equations, 120 (1995), 174-197.

[16] S.J. Cox, M. Ross The maximization of Neumann eigenvalues on convex domains, to appear.

[17] R. Courant, D. Hilbert, Methods of Mathematical Physics, vol. 1 et 2, Wiley, New York, 1953 et 1962.

[18] G. Dal Maso, An introduction to Г-convergence, Birkhäuser, Boston, 1993.

[19] D. Daners, Robin boundary value problems on arbitrary domains, Trans. AMS, $\mathbf{3 5 2}$ (2000), 4207-4236.

[20] R. Dautray and J. L. Lions (ed), Analyse mathématique et calcul numérique, Vol. I and II, Masson, Paris, 1984.

[21] G. FABER, Beweis, dass unter allen homogenen Membranen von gleicher Fläche und gleicher Spannung die kreisförmige den tiefsten Grundton gibt, Sitz. Ber. Bayer. Akad. Wiss. (1923), 169-172. 
[22] M. Flucher, Approximation of Dirichlet eigenvalues on domains with small holes, J. Math. Anal. Appl., 193 (1995), no. 1, 169-199.

[23] E.M. Harrell, P. Kröger, K. Kurata, On the placement of an obstacle or a well so as to optimize the fundamental eigenvalue, to appear in SIAM J. Math. Anal.

[24] A. Henrot, E. Oudet, Le stade ne minimise pas $\lambda_{2}$ parmi les ouverts convexes du plan, C. R. Acad. Sci. Paris Sr. I Math, 332 (2001), no. 4, 275-280.

[25] A. Henrot, E. Oudet, Minimization of the second Dirichlet eigenvalue amongst convex domains, to appear.

[26] A. Henrot, M. Pierre, Optimisation de forme, book to appear.

[27] J. Hersch, The method of interior parallels applied to polygonal or multiply connected membranes, Pacific J. Math., 13 (1963), 1229-1238.

[28] J. Hersch Contraintes rectilignes parallles et valeurs propres de membranes vibrantes, Z. Angew. Math. Phys., 17 (1966) 457-460.

[29] J. Hersch, L.E. PAYne, Extremal principles and isoperimetric inequalities for some mixed problems of Stekloff's type, Z. Angew. Math. Phys., 19 (1968), 802-817.

[30] J. Hersch, L.E. Payne, M.M. Schiffer, Some inequalities for Stekloff eigenvalues, Arch. Rational Mech. Anal., 57 (1975), 99-114.

[31] B. KawOHL, Rearrangements and convexity of level sets in PDE, Lecture Notes in Mathematics, 1150. Springer-Verlag, Berlin, 1985.

[32] B. Kawohl, O. Pironneau, L. Tartar, J.P. Zolsio, Optimal shape design, Lecture Notes in Mathematics, 1740. (Lectures given at the Joint C.I.M./C.I.M.E. Summer School held in Tria, June 1-6, 1998, Edited by A. Cellina and A. Ornelas).

[33] S. Kesavan, On two functionals connected to the Laplacian in a class of doubly connected domains, to appear.

[34] E. KRAHn, Über eine von Rayleigh formulierte Minimaleigenschaft des Kreises, Math. Ann., 94 (1924), 97-100.

[35] E. KRAhn, Über Minimaleigenschaften der Kugel in drei un mehr Dimensionen, Acta Comm. Univ. Dorpat. A9 (1926), 1-44.

[36] A. Melas, On the nodal line of the second eigenfunction of the Laplacian in $\mathbb{R}^{2}, \mathrm{~J}$. Diff. Geometry, 35 (1992) 255-263.

[37] N.S. NADIRASHVILI, Rayleigh's conjecture on the principal frequency of the clamped plate, Arch. Rational Mech. Anal., 129 (1995), 1-10. 
[38] R. Osserman, The isoperimetric inequality, Bull. AMS, 84, no 6 (1978), 1182-1238.

[39] R. Osserman, Isoperimetric inequalities and eigenvalues of the Laplacian, Proceedings of the International Congress of Mathematicians (Helsinki, 1978), pp. 435-442, Acad. Sci. Fennica, Helsinki, 1980.

[40] E. OudET, Some numerical results about minimization problems involving eigenvalues, to appear.

[41] L.E. PAYNE, Isoperimetric inequalities and their applications, SIAM Rev. 9 (1967), 453-488.

[42] L.E. PAYne, Some comments on the past fifty years of isoperimetric inequalities, Inequalities (Birmingham, 1987), 143-161, Lecture Notes in Pure and Appl. Math., 129, Dekker, New York, 1991.

[43] L.E. Payne, G. Pólya, H.F. Weinberger, On the ratio of consecutive eigenvalues, J. Math. Phys., 35 (1956), 289-298.

[44] L.E. Payne,Payne, P.W. Schaefer, Eigenvalue and eigenfunction inequalities for the elastically supported membrane, Z. Angew. Math. Phys., 52 (2001), no. 5, 888-895.

[45] L.E. Payne, H.F. Weinberger, An optimal Poincaré inequality for convex domains, Arch. Rational Mech. Anal. 5 (1960), 286-292.

[46] L.E. Payne, H.F. Weinberger, Some isoperimetric inequalities for membrane frequencies and torsional rigidity, J. Math. Anal. Appl., 2 (1961), 210-216.

[47] G. PóLYA, On the characteristic frequencies of a symmetric membrane, Math. Z. 63 (1955), 331-337.

[48] G. Pólya And G. Szegö, Isoperimetric inequalities in mathematical physics, Ann. Math. Studies, 27, Princeton Univ. Press, 1951.

[49] T. RAssias The isoperimetric inequality and eigenvalues of the Laplacian. Constantin Carathodory: an international tribute, Vol. I, II, 1146-1163, World Sci. Publishing, Teaneck, NJ, 1991.

[50] R. Schoen, S.-T. Yau, Lectures on differential geometry, Conference Proceedings and Lecture Notes in Geometry and Topology, I. International Press, Cambridge, MA, 1994.

[51] J. SerRin, A symmetry problem in potential theory, Arch. Rational Mech. Anal., 43 (1971), 304-318.

[52] J.Simon, Differentiation with respect to the domain in boundary value problems, Num. Funct. Anal. Optimz., 2 (1980) 649-687. 
[53] J. Sokolowski, J.P. Zolesio, Introduction to shape optimization: shape sensitity analysis, Springer Series in Computational Mathematics, Vol. 10, Springer, Berlin 1992.

[54] G. SzeGö, Inequalities for certain eigenvalues of a membrane of given area, J. Rational Mech. Anal. 3 (1954), 343-356.

[55] B.A. Troesch, Elliptical Membranes with smallest second eigenvalue, Math. of Computation, 27-124 (1973) 767-772.

[56] H. F. Weinberger, An isoperimetric inequality for the $N$-dimensional free membrane problem, J. Rational Mech. Anal. 5 (1956), 633-636.

[57] R. Weinstock, Inequalities for a classical eigenvalue problem, J. Rational Mech. Anal., 3 (1954), 745-753.

[58] S.A. Wolf, J.B. Keller, Range of the first two eigenvalues of the Laplacian, Proc. R. Soc. London A, 447 (1994), p. 397-412.

[59] S.-T. YAu Problem section, Seminar on Differential Geometry, pp. 669-706, Ann. of Math. Stud., 102, Princeton Univ. Press, Princeton, N.J., 1982.

[60] S.-T. YAU Open problems in geometry. Differential geometry: partial differential equations on manifolds, (Los Angeles, CA, 1990), 1-28, Proc. Sympos. Pure Math., 54, Part 1, Amer. Math. Soc., Providence, RI, 1993. 